\title{
Is there any influence of elevated diastolic blood pressure on ventricular arrhythmias in hypertensive patients with left ventricular hypertrophy?
}

\author{
Juraj Kunišek ${ }^{1 *}$, Leon Kunišek ${ }^{2}$ \\ ${ }^{1}$ Special Hospital for Medical Rehabilitation, Crikvenica, Croatia \\ ${ }^{2}$ University of Rijeka School of Medicine, University Hospital Centre Rijeka, Rijeka, Croatia
}

Objective: To investigate the prevalence of ventricular arrhythmias in a patient with isolated systolic hypertension (ISHT) and left ventricular hypertrophy (LVH) in comparison to patients with systolic and diastolic hypertension and LVH.

Patients and Methods: 192 (87 men) patients with essential hypertension and LVH were divided into two groups: 98 patients with ISHT and 94 patients with systolic and diastolic hypertension. After discontinuing all medications for a period of 48 hours, the blood pressure was measured, electrocardiography, echocardiography, Holter monitoring and bicycle ergometry were performed. The number of ventricular arrhythmias was recorded. Antihypertensive drugs and the duration of previous treatment were taken into consideration.

Results: Isolated systolic hypertension (systolic blood pressure $>140 \mathrm{mmHg}$ and diastolic blood pressure $<90 \mathrm{mmHg}$ )

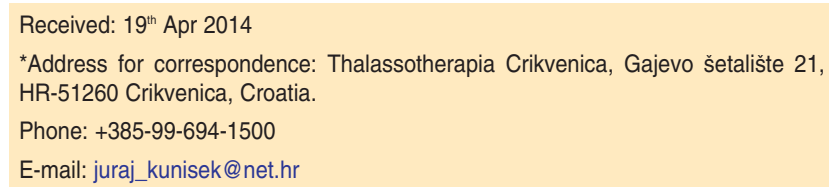

*Address for correspondence: Thalassotherapia Crikvenica, Gajevo šetalište 21, HR-51260 Crikvenica, Croatia.

Phone: +385-99-694-1500

E-mail: juraj_kunisek@net.hr

Table 1. Numerical review of patients.

\begin{tabular}{|l|ccccc|}
\hline \multirow{2}{*}{ No of patients } & \multicolumn{5}{|c|}{ No of patients with } \\
& ISHT & $\%$ & SH+DH & $\%$ & p \\
\hline Total 192 & 98 & $(51)$ & 94 & $(49)$ & NS \\
Man & 47 & $(54)$ & 40 & $(46)$ & \\
Women & 51 & $(49)$ & 54 & $(51)$ & \\
\hline
\end{tabular}

ISHT=isolated systolic hypertension, $\mathrm{SH}=$ systolic hypertension, $\mathrm{DH}=$ diastolic hypertension. was recorded in 98 patients (51\%), 47 men and 51 women (Table 1). The frequency of ventricular arrhythmias did not differ according to Lowns classification categories I-II and IIIIV in patients with and without isolated systolic hypertension (Table 2). There was no difference in the treatment duration between the groups $(p=0.858)$, or in the type of the applied medication. We found $44 \%$ complex ventricular arrhythmias in patients with ISHT. No significant difference was found in the frequency of ventricular arrhythmias between the groups that were observed.

Conclusions: No significant difference was found in the frequency of ventricular arrhythmias in patients with isolated systolic hypertension and LVH in comparison to patients with systolic and diastolic hypertension and LVH. Diastolic blood pressure does not seem to have any influence on the frequency of ventricular arrhythmias in such patients.

KEYWORDS: isolated systolic hypertension, left ventricular hypertrophy, ventricular arrhythmias.

CITATION: Cardiol Croat. 2014;9(5-6):165.

Table 2. Frequency of ventricular arrhythmias in patients with and without isolated systolic hypertension.

\begin{tabular}{|l|cc|cc|c|c|}
\hline & \multicolumn{2}{|c|}{ SH+DH } & \multicolumn{2}{|c|}{ ISHT } & \multirow{2}{*}{ No } & \multicolumn{2}{|c|}{ No } & $\%$ & Total & p \\
\hline Lown I-II & 98 & $(63)$ & 20 & $(56)$ & 118 & \multirow{2}{*}{0.419} \\
\cline { 1 - 5 } Lown III-V & 58 & $(37)$ & 16 & $44)$ & 74 & \\
\hline
\end{tabular}

$\mathrm{SH}=$ systolic hypertension, $\mathrm{DH}=$ diastolic hypertension, ISHT=isolated systolic hypertension. 